\title{
Principios de la gestión financiera en el sector ganadero de una localidad de Venezuela
}

\author{
Principles of financial management in the livestock sector of a town in Venezuela \\ Princípios de gestão financeira no setor pecuário de um município da Venezuela
}

Recibido: octubre 2018

Arbitrado: noviembre 2018

Publicado: enero 2019
《 Eulimar Mosquera

eulimarmos@hotmail.com

ORCID: 0000-0002-8503-6058

Universidad del Zulia. Núcleo Costa Oriental del Lago, Venezuela
RESUMEN

Con el objetivo de identificar los principios de la gestión financiera, se realiza esta investigación desde el enfoque descriptivo, con un diseño no experimental, transeccional y de campo. Para ello se consideró como población, nueve (9) haciendas, tomando como sujetos informantes a nueve (9) administradores de las mismas. Para la recolección de datos se aplicó la técnica de la encuesta, utilizando un cuestionario, conformado por nueve (9) ítems. La validez se hizo bajo el juicio de expertos. Para la confiabilidad, se empleó el Método Alfa de Cronbach, obteniendo un resultado de 0,83. El análisis de los datos se realizó mediante la estadística descriptiva, a través del análisis cuantitativo de frecuencias absolutas y relativas. Evidenciándose muy alta aplicación de la evaluación, control y seguimiento; y alta aplicación de los procesos de planificación y organización.

Palabras clave: Economía; eficacia; eficiencia; gestión financiera; principios
ABSTRACT

RESUMO
In order to identify the principles of financial management, this research is carried out from the descriptive approach, with a non-experimental, cross-sectional and field design. For this, nine (9) haciendas were considered as a population, taking nine (9) administrators of them as informants. For the data collection, the survey technique was applied, using a questionnaire made up of nine (9) items. Validity was made under expert judgment. For reliability, the Cronbach's Alpha Method was used, obtaining a result of 0.83 . Data analysis was performed using descriptive statistics, through the quantitative analysis of absolute and relative frequencies. Evidence of very high application of evaluation, control and monitoring; and high application of planning and organization processes.

Key words: Economy; effectiveness; efficiency; Financial management; beginning
Com o objetivo de identificar os princípios da gestão financeira, esta pesquisa é realizada a partir de uma abordagem descritiva, com um desenho não experimental, transversal e de campo. Para tanto, nove (9) fazendas foram consideradas como uma população, tendo como sujeitos de notificação nove (9) administradores. Para a coleta de dados, foi aplicada a técnica de survey, por meio de um questionário, composto por nove (9) itens. A validade foi feita sob o julgamento de especialistas. Para a confiabilidade, foi utilizado o Método Alpha de Cronbach, obtendo-se um resultado de 0,83. A análise dos dados foi realizada por meio de estatística descritiva, por meio da análise quantitativa de frequências absolutas e relativas. Evidenciou aplicação muito alta de avaliação, controle e monitoramento; e alta aplicação de processos de planejamento e organização.

Palavras-chave: Economia; eficácia; eficiência; gestão financeira; começando 


\section{INTRODUCCIÓN}

$\mathrm{E}$ n Venezuela, el sistema financiero ha venido adquiriendo cada día mayor relevancia por el papel que juega en la política económica orientada al logro de la máxima prosperidad, traducida no solo en aumentar los índices de riqueza sino en un mejoramiento sustancial del nivel de vida (Pico y Pulgar, 2006). El grado de organización y eficiencia del sistema financiero determinará el grado de movilidad y combinación de los recursos disponibles para fines de inversión y por añadidura el grado de utilización óptima o no de dichos recursos.

Por otro lado, el mundo empresarial venezolano se ha caracterizado por la alta presencia de empresas familiares, las cuales deben enfrentar grandes desafíos, representados en constantes cambios de productos y servicios, competidores, ambientes dinámicos, riesgosos, motivo por el cual se ven afectadas. Ante esta situación, para ser competitivas se debe revisar de manera continua los procesos financieros, mejorándolos en la búsqueda de lograr el éxito deseado, en los continuos periodos, esto se traduce en realizar una eficiente gestión financiera.

De esta situación no escapa el sector ganadero, el cual según Nava y otros (2008), enfrenta importantes problemas referidos a la ausencia de infraestructura y de políticas gubernamentales que fomenten esta actividad, inadecuada organización de sus procesos productivos, escasa formación gerencial y carencia de criterios administrativos por parte de quienes manejan las empresas agropecuarias, aunado a un desigual desarrollo de sus unidades productivas.

Acotan que, este sector está conformado por haciendas manejadas con criterio empresarial pero también conformado en una gran proporción, por fincas familiares con baja productividad, que utilizan técnicas rudimentarias y son manejadas con criterio tradicionalista fundamentado en la experiencia de los productores, lo cual afecta la producción, productividad y rentabilidad.

Por lo tanto, a juicio de estos autores, el sector agropecuario venezolano requiere un mayor proceso de desarrollo mediante el cual sus unidades de producción sean manejadas con criterio empresarial, para obtener mayor eficiencia y productividad de cada una de ellas y alcanzar la expansión del sector en su conjunto. Para ello, es necesario conocer mejor la disponibilidad y combinación de los recursos y los procesos financieros y administrativos aplicados, para lograr que los productores agropecuarios lleven una gestión financiera más eficiente de sus recursos, que les permita obtener altos niveles de productividad, competitividad y rentabilidad.

Al respecto, Gómez (2007) afirma que, en este sector existen muchos productores que no llevan contabilidad, ni administran eficientemente sus empresas, esto trae como consecuencia que aun cuando sean rentables, sus propietarios no están en condiciones de conocer cuáles renglones de sus explotaciones generan mayor rendimiento, cuáles son insuficientes y cuáles generan pérdidas; por lo tanto, es necesario establecer un sistema de gestión financiera de fincas que permita conocer el desarrollo de la actividad productiva que se lleva a cabo en ellas. 
Aunado a esto, las nuevas tecnologías, los requerimientos de nuevos insumos, el manejo eficiente de recursos, los estados financieros ajustados por la inflación, la creación de presupuestos de ingresos y egresos con suficiente antelación, entre otros, han llevado a que la administración o gerencia financiera de esta actividad productiva adquiera una gran relevancia.

Aun cuando en el sector ganadero las funciones de gerencia relacionadas con la planificación, organización y dirección son puestas en práctica con frecuencia, se evidencian fallas en lo referente a la función de control y evaluación de resultados, según Bermúdez y Aguirre (2005), debido a la escasez de registros y un inadecuado proceso contable que dificulta el análisis del proceso productivo diario llevado a cabo en estos sistemas.

Específicamente, los ganaderos en el Municipio Mauroa del Estado Falcón sufren esta problemática; en la última década han enfrentado situaciones que se oponen al desarrollo del sector.

Por una parte, estos ganaderos han enfrentado una crisis de eficiencia, bajos niveles de productividad que se reflejan en una disminución de sus niveles de rentabilidad, ausencia de políticas referidas a financiamiento, precios y comercialización de sus productos, y por otra parte, la liberalización del mercado y poca intervención del Estado en la actividad agrícola han agravado el riesgo de disminución del aporte de esta región a la oferta nacional e incrementan los riesgos de crisis económica y social.

Por tal motivo, el objeto de estudio de la investigación esta centrado en analizar la gestión financiera en el sector ganadero en Venezuela, identificando los principios de gestión financiera aplicados.

\section{Principios de la gestión financiera}

Las empresas requieren de recursos humanos, materiales y financieros, así éstas sean pequeñas, medianas o grandes. El papel de las finanzas estará orientado a los recursos financieros, por cuanto el dinero es un recurso para adquirir activos, cancelar obligaciones inmediatas y a largo plazo.

En este sentido, afirma Escribano (2011) que la calidad de la gestión financiera comprenderá cumplir con tres principios: 1) el principio de eficacia, entendido como la consecución de los objetivos, metas y estándares orientados a la satisfacción de necesidades y expectativas del ciudadano; 2) principio de eficiencia, comprendido por la optimización pública en relación a los recursos disponibles e invertidos en su consecución; 3) principio de economía, correspondiendo al uso racional de los recursos.

Por su parte, afirma Frontera (2009) que, algunos de los principios para una buena gestión financiera, serían:

- Hay que saber que toda decisión, acción o inversión en la empresa genera un flujo de caja, el cual se debe estimar lo más realista y conservadoramente posible, para conocer con 
máxima precisión las necesidades de dinero que exige y la rentabilidad que produce. Hasta no disponer y analizar esos datos no se debe ejecutar la decisión

- Es imprescindible llevar un seguimiento de la situación de tesorería con la máxima prontitud, a corto, medio y a largo plazo.

- Estimar y programar las necesidades financieras con suficiente antelación para buscar y comprometer la financiación necesaria.

- Siempre ir por delante y anticiparse a los problemas.

En esta línea del pensamiento, afirma Castán (2005) que, para lograr una eficiente gestión financiera es necesario tener conocimiento claro de cuáles son los objetivos y políticas de la empresa, que por lo general están determinados por los accionistas, que en coordinación con el grupo administrativo le confieren deberes y responsabilidades, delegación de autoridad suficiente orientada a elevar al máximo los intereses de los dueños mediante la maximización de los recursos disponibles y del capital invertido.

Argumenta este autor que, los administradores al ver que sus empresas tienen éxito deben velar por los trabajadores, proveedores, acreedores y en especial por los clientes, que son los que influyen de manera muy significativa sobre el valor de la empresa. Para ello deben valerse de la utilización de los principios económicos (eficacia, eficiencia y economía) que, si bien son generalizaciones, de alguna manera, imprecisas cuantitativamente ya que provienen de la observación del comportamiento social, ofrecen el conocimiento teórico clave para el trabajo del analista financiero.

Adicional a esto, Frontera (2009) afirma que, las actividades de las empresas están influenciadas por el entorno económico, no solamente del país donde están sino también del contexto internacional. El conocimiento de la situación económica del país y de la economía mundial, ayudará al gerente financiero para la toma de decisiones.

Bajo este enfoque, Gitman (2007) considera que dentro de los principios de la gestión financiera el riesgo es uno de los factores más importantes, ya que en el momento que se decide invertir ese exceso de dinero que se posee, ya sea por ahorro o pasadas inversiones, se debe tener en cuenta que se puede optar por dos caminos, el agresivo que es donde se corre mayor riesgo pero al mismo tiempo la inversión es mucho más rentable, o el camino pasivo donde se corre un menor riesgo y se tiene una inversión mucho más estable pero al mismo tiempo poco rentable, teniendo en cuenta el objetivo de acumular ganancias, saberlas mantener y saberlas utilizar

Por ello, afirma este autor, se debe entender que un inversionista racional no está dispuesto a invertir hoy para recibir mañana, ya que debido a factores como la inflación no se pueden comprar las mismas cosas hoy a las que compraría en el futuro con ese mismo valor. Este fenómeno se mide con las tasas de interés, las cuales determinan estas fluctuaciones, de tal manera que el flujo de efectivo es muy importante a la hora de tomar decisiones porque se debe visualizar a la empresa 
con o sin ese producto que se busca introducir, analizando los puntos, identificando así los buenos proyectos.

Con el dinero es que se puede manipular para lograr un beneficio, y así los objetivos planteados por los inversionistas para medir el valor que podría obtener de una inversión en un futuro, logrando estimar si los costos son mayores que los beneficios.

De esta manera, se considera que la gestión financiera será de calidad si cumple con los principios básicos de dicha gestión, asumiendo como principios básicos los referidos por Escribano (2011) y Castán (2005): eficacia, eficiencia y economía. Así mismo, se abordarán cada uno de estos principios asumiéndolos como indicadores que permitirán medir esta dimensión.

\section{Eficacia}

Representada por el grado de cumplimiento de los objetivos y consecución de mayores resultados posibles sin considerar los recursos empleados (Escribano, 2011). Afirma este autor que, todas las empresas, incluso las de menor dimensión y las de reciente creación, deben definir e implementar dinámicas de planificación, organización y control económico-financiero para gestionar sus recursos de forma eficiente y eficaz; dicho de otra manera, la eficacia como principio de la gestión financiera mide los resultados alcanzados en función de los objetivos que se han propuesto.

Al mismo tiempo, Parejo (2007) considera que el estado eficaz, es aquél en que se da un cumplimiento efectivo y satisfactorio de los intereses generales a los que se debe el estado y que justifican la administración, en este caso la financiera. Ese cumplimiento legítimo la acción administrativa.

La administración, o gestión, no sólo se ocupa de determinar las actividades y cumplir con las metas de la organización (eficacia), sino también de hacerlo de la manera más eficiente (organización exitosa). Dentro de esta perspectiva, la eficacia es completar actividades para conseguir las metas con todos los recursos disponibles. Se define como "hacer las cosas correctas".

Por su parte, Andrade (2005) afirma que el término eficaz es un adjetivo utilizado para señalar la capacidad o habilidad de obtener los resultados esperados en determinada situación. El término no sólo es aplicado para personas e individuos, también puede ser eficaz un dispositivo, un tipo de tecnología, un fenómeno natural y diversas cosas, pues no es necesariamente una posibilidad humana. Ser eficaz implica que la metodología de acción que se aplica es la apropiada y, por tanto, la consecuencia será la esperada.

En este orden de ideas, eficaz tiene relación con la idea de efecto ya que es un vínculo directo entre causa o estímulo y efecto. A determinada acción le seguirá siempre un resultado, es decir, un efecto. Cuando ese efecto es el apropiado y buscado, la acción se transforma en una acción eficaz. La eficacia de una acción puede tomarse en cuenta para el desarrollo de estrategias a futuro que permitan obtener similares resultados. 
Ahora bien, Robbins y Coulter (2005) definen la eficacia como el hacer las cosas correctas, es decir, las actividades de trabajo con las que la organización alcanza sus objetivos. Lo principal consiste en definir un objetivo previamente. La eficacia se basa en lo que se llama operacionalización: todo aquello tangible y sirve para cumplir el objetivo que se ha planificado. Estrategias de marketing, planeamiento, establecimiento de métricas, entre otros, pero, sobre todo, buenas ideas, creatividad e innovación.

Los autores citados convergen en que la eficacia está relacionada con el logro de los objetivos, es decir, con la realización de actividades que permita alcanzar las metas establecidas. La eficacia es la medida en que se alcanzan los objetivos o resultados para llegar a alcanzar el éxito en la gestión financiera.

Por lo tanto el término eficaz se utiliza en gran parte de los ámbitos profesionales y empresariales, en los cuales se realiza la aplicación de ciertos tipos de estrategias para la obtención de una gestión eficaz, está directamente vinculada con la obtención de resultados que beneficien a la actividad planeada. Así, la gestión financiera será eficaz cuando los resultados alcanzados estén en función de los objetivos que se han propuesto de la manera más eficiente.

\section{Eficiencia}

La eficiencia, como principio de la gestión financiera, es conseguir los mayores resultados posibles al menor coste posible (Escribano, 2011). La eficiencia económica pone en relieve entonces la relación entre el costo y el valor de lo producido. En otras palabras, la eficiencia financiera es el uso racional de los medios con que se cuenta para alcanzar un objetivo. Se trata de la capacidad de alcanzar objetivos y metas programadas con el mínimo de recursos disponibles y tiempo, logrando su optimización.

En un sentido amplio, Parejo (2007) afirma que la eficiencia de una gestión se evalúa en términos de relación de medios afines. La palabra eficiencia tiene su origen en el término latino efficientia y refiere a la capacidad de disponer de alguien o de algo para conseguir un efecto determinado, también suele ser equiparado con el de acción, fuerza o producción. Para Chiavenato (2006) significa utilización correcta de los recursos (medios de producción) disponibles. Puede definirse mediante la ecuación $E=P / R$, donde $P$ son los productos resultantes y $R$ los recursos utilizados.

Chiavenato (2006) también la define como la relación entre los recursos utilizados en un proyecto y los logros conseguidos con el mismo, la eficiencia se da cuando se utilizan menos recursos para lograr un mismo objetivo, o, al contrario, cuando se logran más objetivos con los mismos o menos recursos.

Por otra parte, Koontz y Weihrich (2005) plantean que es el logro de las metas con la menor cantidad de recursos. La eficiencia está vinculada a utilizar medios disponibles de manera racional para llegar a una meta. Se trata de la capacidad de alcanzar un objetivo fijado con anterioridad en el menor tiempo posible, y con el mínimo uso posible de los recursos, lo que supone una 
optimización. En este sentido, para Robbins y Coulter (2005) consiste en obtener mayores resultados con la mínima inversión.

Ahora bien, Castán (2005) define eficiencia, desde lo más general, como la virtud y facultad para lograr un efecto determinado; en economía lo define como el empleo de medios en tal forma que satisfagan un máximo cuantitativo o cualitativo de fines o necesidades humanas, en otras palabras, consiste en el buen uso de los recursos.

En el ámbito de la gestión financiera, la define como lograr lo mayor posible con aquello que se cuenta, si un grupo humano dispone de un determinado número de insumos que son utilizados para producir bienes o servicios, eficiente será aquel grupo que logre el mayor número de bienes o servicios utilizando el menor número de insumos que le sea posible, para llevarlo a una gestión financiera plena. Continua afirmando que, eficiencia se emplea para relacionar los esfuerzos frente a los resultados que se obtengan; a mayores resultados, mayor eficiencia. Si se obtienen mejores resultados con menor gasto de recursos o menores esfuerzos, se habrá incrementado la eficiencia.

De modo que, eficiencia se refiere a la producción de bienes o servicios que la sociedad valora más, al menor costo social posible. Es el cociente entre resultados obtenidos y el valor de los recursos empleados. La eficiencia no es un valor absoluto que se alcanza por sí mismo, sino que se determina por comparación con los resultados obtenidos por terceros, quienes actúan en situaciones semejantes a las que se desean analizar.

Se debe tener presente que eficiencia es la capacidad de hacer correctamente las cosas, lograr resultados de acuerdo a la inversión o esfuerzo que se realice. Eficacia es la capacidad de escoger los objetivos apropiados. Un administrador financiero eficaz y eficiente será aquel que selecciona los objetivos correctos para trabajar en el sentido de alcanzarlos con el menor costo posible.

\section{Economía}

La economía en la gestión financiera consiste en hacer las cosas al menor coste posible, lo que implica que los resultados se dispongan en el momento adecuado, la cantidad adecuada y la calidad requerida. También se ocupa de la manera que se administran recursos escasos, con objeto de producir bienes y servicios y distribuirlos para su consumo entre los miembros de la sociedad (Escribano, 2011). En este punto, Samuelson y Nordhaus (2005) definen la economía como: el estudio de la manera en que las sociedades utilizan los recursos escasos para producir mercancías valiosas y distribuirlas entre los diferentes individuos.

En efecto, es la forma en la que la empresa puede manejar sus recursos y servicios, ofreciendo una visión competitiva frente al mercado. Se vale de varias disciplinas científicas que permiten llevar a cabo dicha labor. Es una forma de aplicar economía en el ámbito de una empresa y deben tenerse en cuenta para su buen funcionamiento valores externos como índices de la bolsa, demanda de mercado y otras variables.

Mientras que para Bouza (2006), economía se refiere a los términos y condiciones bajo los cuales se adquieren y utilizan los recursos humanos, financieros y materiales, tanto en cantidad y 
calidad apropiadas, como al menor costo posible, y de manera oportuna. De tal forma que, la economía es el estudio de cómo los individuos transforman los recursos naturales en productos y servicios finales que puedan ser utilizados.

En este sentido, para Castán (2005), la economía es un aspecto clave en los principios de gestión financiera debido a que estudia los modos a proceder para la administración y gestión de unos recursos limitados y disponibles, con objeto de obtener bienes o servicios que satisfagan las necesidades de las personas u organizaciones, definiendo la economía como la ciencia o rama del pensamiento humano por la cual la exposición de una o varias teorías permite predecir el comportamiento del objeto que se estudia.

La economía engloba procesos de extracción y obtención de materias primas, procesos de transformación y producción, así como la comercialización y distribución de los productos y servicios, mediante unos recursos limitados. La principal característica de la economía es que cuenta con unos recursos limitados, en el caso que los recursos fueran ilimitados no existiría la economía como tal ni como se conoce, dado a que las necesidades serían satisfechas al instante sin ningún tipo de esfuerzo. Es por ello que este concepto es fundamental para lograr una buena gestión en cualquier actividad llevada a cabo.

\section{MÉTODO}

$\mathrm{L}$ a presente investigación se consideró descriptiva, debido a que su propósito fundamental consistió en describir el comportamiento de la variable gestión financiera en el sector ganadero, resaltando el carácter asumido en la dimensión principios de la gestión financiera, con un diseño no experimental, transeccional y de campo.

La población estuvo conformada por nueve (9) haciendas activas, adscritas a la Asociación de Ganaderos en el municipio Mauroa del estado Falcón en Venezuela, siendo los principales sujetos informantes los nueve (9) administradores que llevan la gestión financiera de dichas haciendas. Se destaca que, el criterio de selección de las haciendas fue el estar constituidas formalmente desde el año 1990. En este caso, y debido al tamaño finito de la población, en la presente investigación no se aplicó método de muestreo, se consideró y seleccionó el $100 \%$ de la misma como muestra representativa, enmarcándose en un censo poblacional; es decir, 9 sujetos.

Para la recolección de datos se aplicó la técnica de la encuesta, utilizando un cuestionario, conformado por nueve (9) ítems, con escala de respuesta de frecuencia: nunca, casi nunca, a veces, casi siempre y siempre. La validez se hizo bajo el juicio de cinco (5) expertos. Para la confiabilidad, se empleó el Método Alfa de Cronbach, obteniendo un resultado de 0,83, lo cual indicó muy alta confiabilidad, implicando que el instrumento era apto para su aplicación.

El análisis de los datos se realizó mediante la estadística descriptiva, a través del análisis cuantitativo de frecuencias absolutas y relativas, el cual se presentó en tablas para describir y ordenar debidamente las respuestas de los ítems. A tal efecto, para el análisis de las frecuencias se 
consideró conveniente fijar un baremo de interpretación el cual se recoge en la tabla 1, en el mismo se mostró el rango en donde pueden darse los resultados con base a la suma de las respuestas más positivas, siempre y casi siempre $(S+C s)$, la categoría asignada, así como la descripción de la misma.

Tabla 1. Interpretación del porcentaje de la distribución de frecuencia

\begin{tabular}{lll}
\hline RANGO & CATEGORÍA & INTERPRETACión \\
\hline $75 \% \leq \mathrm{S}+\mathrm{CS}<100 \%$ & $\begin{array}{l}\text { Muy alta aplicación } \\
\text { Alta fortaleza }\end{array}$ & $\begin{array}{l}\text { Muy alta aplicación del ítem, indicador, } \\
\text { dimensión y variable. Implica alta fortaleza para } \\
\text { las haciendas estudiadas. }\end{array}$ \\
$50 \% \leq \mathrm{S}+\mathrm{CS}<75 \%$ & $\begin{array}{l}\text { Alta aplicación } \\
\text { Leve fortaleza }\end{array}$ & $\begin{array}{l}\text { Alta aplicación del ítem, indicador, dimensión y } \\
\text { variable. Implica leve fortaleza para las } \\
\text { haciendas estudiadas. }\end{array}$ \\
& $\begin{array}{l}\text { Baja aplicación } \\
\text { Leve debilidad }\end{array}$ & $\begin{array}{l}\text { Baja aplicación del ítem, indicador, dimensión y } \\
\text { variable. Implica leve debilidad para las } \\
\text { haciendas estudiadas. }\end{array}$ \\
$0 \% \leq \mathrm{S}+\mathrm{CS}<25 \%$ & Muy baja aplicación \\
& Alta debilidad & $\begin{array}{l}\text { Muy baja aplicación del ítem, indicador, } \\
\text { dimensión y variable. Implica alta debilidad para } \\
\text { las haciendas estudiadas. }\end{array}$ \\
\hline
\end{tabular}

\section{RESULTADOS}

$\mathrm{E}$

n la tabla 2 se muestran los resultados para el indicador eficacia, observándose un promedio de frecuencias de $85,19 \%$, indicando que siempre y casi siempre, a criterio de los encuestados, la eficacia como principio de la gestión financiera se aplica con muy alta frecuencia, otorgándole la categoría de muy alta aplicación, implicando a su vez una alta fortaleza para las haciendas bajo estudio. 
Tabla 2. Indicador: Eficacia

\begin{tabular}{ll}
\hline $\begin{array}{l}\text { Variable } \\
\text { Dimensión }\end{array}$ & $\begin{array}{l}\text { Gestión financiera } \\
\text { Principios de la gestión financiera }\end{array}$ \\
\hline
\end{tabular}

\section{Ítems del indicador}

1. Se genera un cumplimiento efectivo y satisfactorio de los intereses.

2. Se tiene la capacidad de obtener los resultados esperados.

3. La administración da cumplimiento al objetivo que se ha planificado.

\begin{tabular}{|c|c|c|c|c|c|c|}
\hline \multirow[b]{2}{*}{ Alternativas } & \multicolumn{2}{|l|}{1} & \multicolumn{2}{|l|}{2} & \multicolumn{2}{|l|}{3} \\
\hline & FA & FR (\%) & FA & FR (\%) & FA & FR (\%) \\
\hline Siempre & 7 & 77,78 & 5 & 55,56 & 5 & 55,56 \\
\hline Casi siempre & 2 & 22,22 & 2 & 22,22 & 2 & 22,22 \\
\hline Algunas veces & 0 & 0,00 & 2 & 22,22 & 2 & 22,22 \\
\hline Casi nunca & 0 & 0,00 & 0 & 0,00 & 0 & 0,00 \\
\hline Nunca & 0 & 0,00 & 0 & 0,00 & 0 & 0,00 \\
\hline Total & 9 & 100 & 9 & 100 & 9 & 100 \\
\hline \multirow[t]{2}{*}{$S+C s$} & \multicolumn{2}{|c|}{$100 \%$} & \multicolumn{2}{|c|}{$77,78 \%$} & \multicolumn{2}{|c|}{$77,78 \%$} \\
\hline & \multicolumn{2}{|c|}{ Muy alta aplicación } & \multicolumn{2}{|c|}{ Muy alta aplicación } & \multicolumn{2}{|c|}{ Muy alta aplicación } \\
\hline Categoría & \multicolumn{2}{|c|}{ Alta fortaleza } & \multicolumn{2}{|c|}{ Alta fortaleza } & \multicolumn{2}{|c|}{ Alta fortaleza } \\
\hline \multicolumn{7}{|c|}{ Promedio Indicador } \\
\hline & \multicolumn{6}{|c|}{$85,19 \%$} \\
\hline Categoría & \multicolumn{6}{|c|}{ Muy alta aplicación / Alta fortaleza } \\
\hline
\end{tabular}

De tal manera, le confirieron muy alta aplicación a todas las actividades medidas por los ítems utilizados, referidos a si: generan un cumplimiento efectivo y satisfactorio de los intereses, tienen capacidad de obtener los resultados esperados y la administración da cumplimiento al objetivo que se fue planificado, donde el $100 \%$ en el primer caso, y el $77,78 \%$ en los otros dos casos, de los encuestados respondieron en las opciones positivas $(S+C S)$, implicando alta fortaleza para las haciendas estudiadas en lo concerniente a estos aspectos.

Estos resultados coincidieron, con lo expuesto por Parejo (2007) quien considera que el estado eficaz, es aquél en que se da un cumplimiento efectivo y satisfactorio de los intereses generales a los que se debe el estado, y que justifican la administración en este caso la financiera. Ese cumplimiento legítimo la acción administrativa. Dentro de esta perspectiva, la eficacia es completar actividades para conseguir las metas con todos los recursos disponibles. Se define como "hacer las cosas correctas".

De acuerdo a lo evidenciado, se puede decir, que en las haciendas estudiadas se otorgaron al principio eficacia, en la gestión financiera, rol clave que debe adjudicársele, ya que la eficacia de la gestión financiera se da cuando los resultados alcanzados estén en función de los objetivos que se han propuesto de la manera más eficiente; y en virtud de los resultados, esta etapa posee muy alta aplicación. 
Otro indicador considerado en la dimensión bajo análisis, lo constituye la eficiencia. En la Tabla 3 se muestran los resultados obtenidos del trabajo de campo. Se determinó que la eficiencia, como principio de la gestión financiera, obtuvo una categoría de alta aplicación al arribar a un promedio de frecuencias de 51,85\%, implicando una leve fortaleza a este respecto en la gestión financiera que llevan a cabo las haciendas en el sector ganadero del municipio Mauroa estado Falcón en Venezuela.

Tabla 3. Eficiencia

\begin{tabular}{ll}
\hline Variable & Gestión financiera \\
Dimensión & Principios de la gestión financiera \\
\hline
\end{tabular}

\section{Ítems del indicador}

4. Están en capacidad de disponer de la utilización correcta de los recursos.

5. Se logra el objetivo determinado con la menor cantidad de recursos.

6. Su nivel de rentabilidad varía de acuerdo al nivel de inventario.

\begin{tabular}{|c|c|c|c|c|c|c|c|}
\hline \multirow[b]{2}{*}{ Alternativas } & \multicolumn{3}{|l|}{4} & \multicolumn{2}{|l|}{5} & \multicolumn{2}{|l|}{6} \\
\hline & FA & FR (\%) & & FA & FR (\%) & FA & FR (\%) \\
\hline Siempre & 3 & 33,33 & & 3 & 33,33 & 0 & 0,00 \\
\hline Casi siempre & 2 & 22,22 & & 4 & 44,44 & 2 & 22,22 \\
\hline Algunas veces & 4 & 44,44 & & 2 & 22,22 & 7 & 77,78 \\
\hline Casi nunca & 0 & 0,00 & & 0 & 0,00 & 0 & 0,00 \\
\hline Nunca & 0 & 0,00 & & 0 & 0,00 & 0 & 0,00 \\
\hline Total & 9 & 100 & & 9 & 100 & 9 & 100 \\
\hline \multirow[t]{2}{*}{$S+C s$} & \multicolumn{3}{|c|}{$55,56 \%$} & \multicolumn{2}{|c|}{$77,78 \%$} & \multicolumn{2}{|c|}{$22,22 \%$} \\
\hline & \multicolumn{2}{|c|}{$\begin{array}{l}\text { Alta aplicación } \\
\text { fortaleza }\end{array}$} & Leve & \multicolumn{2}{|c|}{$\begin{array}{l}\text { Muy alta aplicación } \\
\text { Alta fortaleza }\end{array}$} & \multicolumn{2}{|c|}{$\begin{array}{l}\text { Muy baja aplicación } \\
\text { Alta debilidad }\end{array}$} \\
\hline \multicolumn{8}{|l|}{ Promedio } \\
\hline Indicador & \multicolumn{7}{|c|}{$51,85 \%$} \\
\hline Categoría & \multicolumn{7}{|c|}{ Alta aplicación / Leve fortaleza } \\
\hline
\end{tabular}

Al detalle, al consultarles si se logra el objetivo determinado con la menor cantidad de recursos el $77,78 \%$ respondieron en las categorías positivas $(S+C s)$, confiriéndole a esta actividad muy alta aplicación e implicando alta fortaleza según el baremo. Mientras que para el 55,56\% siempre y casi siempre están en capacidad de disponer de la utilización correcta de los recursos, asignándole alta aplicación por ende se convierte en una leve fortaleza. Ahora bien, solo el $22,22 \%$ de los encuestados respondieron de forma positiva en cuanto a si su nivel de rentabilidad varía de acuerdo al nivel de inventario, reseñándola como de muy baja aplicación y alta debilidad.

Las situaciones precedentes, de alta aplicación de la eficiencia, validan a Escribano (2011), para quien la eficiencia, como principio de la gestión financiera, persigue conseguir los mayores resultados posibles al menor coste posible. Se trata de la capacidad de alcanzar objetivos y metas programadas con el mínimo de recursos disponibles y tiempo, logrando su optimización. 
Por lo tanto, las haciendas bajo estudio se coordinaron de manera efectiva, en su proceso de gestión financiera de acuerdo al desarrollo efectivo de sus actividades, presentando fortalezas. No obstante, poseían oportunidades de mejora en cuanto al uso de sus niveles de inventario. Se debe tener presente que la eficiencia es alcanzar los objetivos por medio de la elección de alternativas que permita suministrar un mayor beneficio.

Siguiendo con el recorrido de los resultados, se tienen los obtenidos para el indicador economía de la dimensión principios de la gestión financiera, los cuales se resumen en la tabla 4, donde se evidenció que el mismo fue catalogado con muy alta aplicación, constituyéndose en alta fortaleza para las haciendas estudiadas, mostrado por un promedio de frecuencias relativas, en las opciones siempre y casi siempre, de $81,48 \%$.

Tabla 4. Economía

\begin{tabular}{|c|c|c|c|c|c|c|}
\hline Variable & \multicolumn{6}{|c|}{ Gestión financiera } \\
\hline Dimensión & \multicolumn{6}{|c|}{ Principios de la gestión financiera } \\
\hline \multicolumn{7}{|c|}{ Ítems del indicador } \\
\hline \multicolumn{7}{|c|}{ 7. Determinan condiciones para el uso de los recursos. } \\
\hline \multicolumn{7}{|c|}{ 8. Utilizan los recursos adecuados según los parámetros. } \\
\hline \multicolumn{7}{|c|}{ 9. Se formulan criterios de proceder para la administración. } \\
\hline \multirow[b]{2}{*}{ Alternativas } & \multicolumn{2}{|l|}{7} & \multicolumn{2}{|l|}{8} & \multicolumn{2}{|l|}{9} \\
\hline & FA & FR (\%) & FA & FR (\%) & FA & FR (\%) \\
\hline Siempre & 5 & 55,56 & 0 & 0,00 & 0 & 0,00 \\
\hline Casi siempre & 4 & 44,44 & 9 & 100 & 4 & 44,44 \\
\hline Algunas veces & 0 & 0,00 & 0 & 0,00 & 5 & 55,56 \\
\hline Casi nunca & 0 & 0,00 & 0 & 0,00 & 0 & 0,00 \\
\hline Nunca & 0 & 0,00 & 0 & 0,00 & 0 & 0,00 \\
\hline Total & 9 & 100 & 9 & 100 & 9 & 100 \\
\hline \multirow[t]{2}{*}{$\mathbf{S}+\mathbf{C s}$} & \multicolumn{2}{|c|}{$100 \%$} & \multicolumn{2}{|c|}{$100 \%$} & \multicolumn{2}{|c|}{$44,44 \%$} \\
\hline & \multicolumn{2}{|c|}{ Muy alta aplicación } & \multicolumn{2}{|c|}{ Muy alta aplicación } & \multicolumn{2}{|c|}{ Baja aplicación } \\
\hline Categoría & \multicolumn{2}{|c|}{ Alta fortaleza } & \multicolumn{2}{|c|}{ Alta fortaleza } & \multicolumn{2}{|c|}{ Leve debilidad } \\
\hline \multicolumn{7}{|l|}{ Promedio } \\
\hline Indicador & \multicolumn{6}{|c|}{$81,48 \%$} \\
\hline Categoría & \multicolumn{6}{|c|}{ Muy alta aplicación / Alta fortaleza } \\
\hline
\end{tabular}

Al detalle se observó para el $100 \%$ de los encuestados siempre y casi siempre, que determinan condiciones para el uso de los recursos y utilizan los recursos adecuados según los parámetros, indicando muy alta aplicación de estas actividades, por ende, representan altas fortalezas, según el baremo diseñado. Mientras que para el $44,44 \%$ siempre y casi siempre se formulan criterios de proceder para la administración, indicando baja aplicación con leve debilidad en este aspecto. 
Los resultados de muy alta aplicación validan, de muy alta manera, lo postulado por Castán (2005), para quien la economía es un aspecto clave en los principios de gestión financiera, debido a que estudia los modos a proceder para la administración y gestión de unos recursos limitados y disponibles, con objeto de obtener bienes o servicios que satisfagan las necesidades de las personas u organizaciones, definiendo la economía como la ciencia o rama del pensamiento humano por la cual la exposición de una o varias teorías permite predecir el comportamiento del objeto que se estudia.

De esta manera, la economía debe contar con recursos limitados, en el caso que los recursos fueran ilimitados no existiría la economía como tal ni como se conoce, dado a que las necesidades serían satisfechas al instante sin ningún tipo de esfuerzo. Es por ello que este concepto es fundamental para lograr una buena gestión en cualquier actividad llevada a cabo.

Ahora bien, desde el punto de vista de la dimensión principios de la gestión financiera, el valor promedio de frecuencias que se obtuvo en sus indicadores, se presentó en la tabla 5,en la suma de las opciones siempre y casi siempre, fue de $72,84 \%$ ubicándose en el rango $50 \% \leq \mathrm{S}+\mathrm{CS}<75 \%$ con una interpretación de alta aplicación e implicando leve fortaleza, lo cual traduce que los principios de la gestión financiera, en las haciendas analizadas, posee alta aplicación en la gestión financiera que desarrollan dichas haciendas, confiriéndoles a éstas una leve fortaleza.

Tabla 5. Principios de la gestión financiera

\begin{tabular}{lll}
\hline $\begin{array}{l}\text { Variable: Gestión financiera } \\
\text { INDICADOR }\end{array}$ & S + Cs & CATEGORÍAS \\
\hline Eficacia & $85,19 \%$ & Muy alta aplicación / Alta fortaleza \\
Eficiencia & $51,85 \%$ & Alta aplicación / Leve fortaleza \\
Economía & $81,48 \%$ & Muy alta aplicación / Alta fortaleza \\
RESUMEN DIMENSIÓN & $\mathbf{7 2 , 8 4 \%}$ & Alta aplicación / Leve fortaleza \\
\hline
\end{tabular}

Los resultados demostraron que, en las haciendas estudiadas, la eficacia y economía como principios de la gestión financiera arriban a la categoría de muy alta aplicación, según la opinión de los encuestados; mientras que la eficiencia muestra alta aplicación siendo una leve fortaleza, aun cuando, cabe acotar, que arriba a esta categoría, pero en los niveles bajos de dicha categoría en el baremo.

Lo precedente, valida la teoría que sustenta esta investigación. En específico, es coincidente con Escribano (2011), cuando afirma que la calidad de la gestión financiera comprenderá cumplir con tres principios: 1) el principio de eficacia, entendido como la consecución de los objetivos, metas y estándares orientados a la satisfacción de necesidades y expectativas del ciudadano; 2) principio de eficiencia, comprendido por la optimización pública en relación a los recursos disponibles e invertidos en su consecución; 3) principio de economía, correspondiendo al uso racional de los recursos. 
De igual manera, se validó lo expresado por Castán (2005) quien asegura que, para lograr una eficiente gestión financiera es necesario tener conocimiento claro de cuáles son los objetivos y políticas de la empresa, que por lo general están determinados por los accionistas, que en coordinación con el grupo administrativo le confieren deberes y responsabilidades, delegación de autoridad suficiente orientada a elevar al máximo los intereses de los dueños mediante la maximización de los recursos disponibles y del capital invertido.

De esta manera se deduce que en las haciendas del sector ganadero del municipio Mauroa estado Falcón en Venezuela, los principios de la gestión financiera se cumplieron con alta frecuencia, ya que han sido declarados con alta aplicación, otorgándoles leves fortalezas en su gestión financiera. Por lo que se consideró que la gestión financiera es de calidad dado que cumplió con los principios básicos de dicha gestión.

\section{CONCLUSIONES}

$\mathrm{E}$ n lo que concierne al objetivo, donde se identificaron los principios de la gestión financiera aplicados en el sector ganadero del municipio Mauroa estado Falcón, se concluye que estos principios muestran alta aplicación como parte de la gestión financiera analizada, lo cual les confiere una leve fortaleza a las haciendas estudiadas. A su vez se pudo observar que la eficacia y economía como principios de la gestión financiera arribaron a la categoría de muy alta aplicación, mientras que la eficiencia muestra alta aplicación siendo una leve fortaleza, aun cuando, cabe acotar, que arriba a esta categoría, pero en los niveles bajos de dicha categoría en el baremo.

Así las cosas, se tienen que revisar aspectos que muestran oportunidades de mejora tanto en la eficiencia que muestra una actividad con alta aplicación, como por supuesto en el principio de economía que muestra una actividad con baja aplicación. En ambos casos, se acota que se debe:

- Velar porque se desarrolle la capacidad de disponer de la utilización correcta de los recursos.

- Garantizar que su nivel de rentabilidad varíe de acuerdo al nivel de inventario.

- Asegurar que se formulen criterios de proceder para la administración.

\section{REFRENCIAS}

Andrade, S. (2005). Diccionario de economía. Editorial Andrade. Granada-España

Bermúdez, A. y Aguirre, J. (2005). Gerencia y aspectos técnico-económicos de los sistemas De ganadería de doble propósito. Universidad del Zulia. Editorial Astro Data. Maracaibo. Venezuela
Bouza, S. (2006). Auditoria operativa y administrativa. Editorial Espasa Calpe. España

Castán, J. (2005). Fundamentos y aplicaciones de la gestión financiera de la empresa. Editorial Pirámide. Madrid. España 
Chiavenato, I. (2006). Introducción a la teoría general de la administración. Cuarta edición. Editorial McGraw-Hill. México

Escribano, G. (2011). Gestión financiera. Tercera edición. Ediciones Paraninfo. España

Frontera, D. (2009). El arte financiero para dirigir empresas. Ed. Almazara. México

Gitman, L. (2007). El futuro de los negocios. Editorial Thompson. 11va. Edición. México

Gómez, F. (2007). Contabilidad ganadera en Venezuela. Ediciones Fragor. Venezuela

Koontz, H. y Weihrich, H. (2005). Administración. Una perspectiva global. 12a. Edición. Editorial McGrawHill Interamericana. México

Nava, M., Urdaneta, F. y Casanova, A. (2008). Gerencia y productividad en sistemas ganaderos de doble propósito. Revista Venezolana de Gerencia (RVG). Año 13. N. ${ }^{\circ} 43$, Universidad del Zulia (LUZ). ISSN 1315-9984. Págs. 468 - 491. Venezuela
Parejo, A. (2007). La eficacia administrativa y la calidad total de los servicios públicos. El derecho administrativo en el umbral del siglo XXI, Homenaje al Prof. Dr. Don Ramón Martin Mateo, T, II, Tirant lo Blanch. Valencia. Venezuela

Pico, G. y Pulgar, A. (2006). Gestión Financiera y Participación en el Mercado de Capitales a Través del Asesor de Inversiones. Revista Actualidad Contable FACES. Año 9 N. ○ 13, Julio-Diciembre 2006. Mérida. Venezuela. (págs. 128-138)

Robbins, S. y Coulter, M. (2005). Administración. Octava Edición. Pearson Educación. México

Samuelson, P. y Nordhaus, W. (2005). Economía. Décimo séptima edición. Editorial McGraw-Hill. México 